\title{
Interlaboratory agreement in the monitoring of unfractionated heparin using the anti-factor Xa-correlated activated partial thromboplastin time
}

\author{
A. CUKER, * B. PTASHKIN, †B.A. KONKLE, ${ }^{\dagger} \dagger$ S. W. PIPE, $\ddagger$ H. C. WHINNA, §X. L. ZHENG, † \\ D. B. CINES*† and E. S. POLLAK† \\ *Department of Medicine and; †Departments of Pathology and Laboratory Medicine, University of Pennsy/vania, Philadelphia, PA; $\ddagger D e p a r t m e n t s$ \\ of Pediatrics and Pathology, University of Michigan, Ann Arbor, MI; §Department of Pathology, University of North Carolina, Chapel Hill, NC; \\ and Department of Pathology, Children's Hospital of Philadelphia, Philadelphia, PA, USA
}

To cite this article: Cuker A, Ptashkin B, Konkle BA, Pipe SW, Whinna HC, Zheng XL, Cines DB, Pollak ES. Interlaboratory agreement in the monitoring of unfractionated heparin using the anti-factor Xa-correlated activated partial thromboplastin time. J Thromb Haemost 2009; 7: 80-6.

Summary. Background: In an effort to improve interlaboratory agreement in the monitoring of unfractionated heparin (UFH), the College of American Pathologists (CAP) recommends that the therapeutic range of the activated partial thromboplastin time (APTT) be defined in each laboratory through correlation with a direct measure of heparin activity such as the factor Xa inhibition assay. Whether and to what extent this approach enhances the interlaboratory agreement of UFH monitoring has not been reported. Objectives: We conducted a crossvalidation study among four CAP-accredited coagulation laboratories to compare the interlaboratory agreement of the anti-FXa-correlated APTT with that of the traditional 1.5-2.5 times the midpoint of normal (1.5-2.5:control) method for defining the therapeutic APTT range. Patients and methods: APTT and FXa inhibition assays were performed in each laboratory on plasma samples from 44 inpatients receiving UFH. Results: Using the anti-FXa-correlation method, there was agreement among all four laboratories as to whether a sample was subtherapeutic, therapeutic or supratherapeutic in seven $(16 \%)$ patient samples. In contrast, consensus was achieved in $23(52 \%)$ samples when the 1.5-2.5:control method was employed. Conclusions: The anti-FXa-correlation method does not appear to enhance interlaboratory agreement in UFH monitoring as compared with the traditional 1.5-2.5:control method. Adoption of the anti-FXa-correlation method produces considerable disparity in UFH dosing decisions among different centers, although the clinical impact of this disparity is not known.

Correspondence: Adam Cuker, Penn Presbyterian Medical Center, MAB 106, 51 N. 39th St, Philadelphia, PA 19104, USA.

Tel.: + 1215662 9273; fax: + 12152434621 .

E-mail: adam.cuker@uphs.upenn.edu

Received 11 July 2008, accepted 30 October 2008
Keywords: activated partial thromboplastin time, antifactor Xa, factor Xa inhibition assay, unfractionated heparin.

\section{Introduction}

Intravenously administered unfractionated heparin (UFH) is a mainstay of inpatient anticoagulation therapy for a variety of clinical indications. Because the anticoagulant response to UFH varies among individuals, the standard of care is to monitor UFH and make dose adjustments on the basis of the results of coagulation testing. Nevertheless, evidence for this practice is weak and is based on a rabbit model of thrombosis [1] and a post hoc subgroup analysis of an observational clinical study [2], which demonstrated that UFH therapy prolonging the activated partial thromboplastin time (APTT) to 1.5-2.5 times the midpoint of the control range (1.5-2.5:control) was associated with a reduction in the risk of recurrent thrombosis. On the basis of this evidence, use of the APTT to monitor UFH with a therapeutic range of 1.5-2.5:control became standard practice.

The wisdom of a so-called fixed ratio method for defining the therapeutic APTT range was later questioned when it was appreciated that various APTT reagent-coagulometer combinations differ, sometimes markedly, in their dose response to UFH [3-8]. With over 300 such combinations in use in different laboratories by 1998 [9], interlaboratory variation in UFH monitoring emerged as a major clinical concern.

To address this concern, the College of American Pathologists (CAP) [9] and the American College of Chest Physicians (ACCP) [10] adopted guidelines recommending that clinical laboratories define the therapeutic APTT range through correlation with a direct assay of heparin activity such as protamine titration or factor Xa inhibition (anti-FXa-correlation method). Whether such an approach enhances interlaboratory agreement in UFH laboratory monitoring has not been reported. We undertook a cross-validation study among 
four CAP-accredited laboratories to determine whether the anti-FXa-correlation method improves upon the interlaboratory variation observed with the 1.5-2.5:control method in the monitoring of UFH.

\section{Methods}

\section{Patient samples}

Samples from 44 adult inpatients at a single academic medical center who were receiving UFH for a variety of clinical indications were selected for analysis. All patients had a normal APTT and prothrombin time prior to initiation of UFH therapy, and none was on concomitant warfarin or other medications reported to affect coagulation testing. The study protocol was reviewed and approved by the institutional review board of the center from which samples were collected.

\section{Laboratory assays}

Venous blood was collected in 4.5-mL evacuated specimen tubes (Becton Dickinson, Franklin Lakes, NJ, USA) containing 3.2\% sodium citrate. The samples were centrifuged at $1900 \mathrm{~g}$ for $10 \mathrm{~min}$ at $4{ }^{\circ} \mathrm{C}$. The plasma was then transferred to a microcentrifuge tube (Eppendorf, Hamburg, Germany) and spun again at room temperature at $13000 \mathrm{~g}$. Aliquots of the plasma were transferred to clean polypropylene tubes (Fisher Scientific, Pittsburgh, PA, USA), capped, and stored at $-70^{\circ} \mathrm{C}$. Frozen aliquots from each of the 44 samples were sent on dry ice to specialized coagulation laboratories at three other major academic medical centers. APTT and FXa inhibition assays were performed on the samples at each of the four laboratories, in accordance with their standard operating procedures. The reagent-instrument combinations employed by each laboratory are listed in Table 1.

\section{Simulated shipment}

In an effort to simulate the shipping process, 10 samples were packed and stored on dry ice for $24 \mathrm{~h}$ in laboratory A. APTT and FXa inhibition assays were performed on each sample

before and after shipment, in order to evaluate the effect of transport on test variability.

\section{Statistical analysis}

Laboratory results within and among laboratories were compared by means of linear regression and tabulation of the coefficient of determination $\left(R^{2}\right)$. Studentized residuals were calculated to detect outliers. All analyses were carried out using NCSS 2007 (NCSS; Kaysville, UT, USA).

\section{Results}

\section{Intralaboratory correlation and derivation of therapeutic ranges}

Modest to poor correlation between the APTT and FXa inhibition assays was observed in each of the four laboratories. The coefficients of determination $\left(R^{2}\right)$ ranged from 0.1962 to 0.6964 (Fig. 1). When the single outlier most affecting the $R^{2}$ at each laboratory was excluded from the analysis, the $R^{2}$ improved to $0.6296,0.7636,0.2708$ and 0.6834 in laboratories $\mathrm{A}, \mathrm{B}, \mathrm{C}$ and $\mathrm{D}$, respectively.

In accordance with CAP guidelines, the anti-FXa-derived therapeutic APTT range was defined by linear regression corresponding to a range of anti-FXa activity of $0.3-$ 0.7 units $\mathrm{mL}^{-1}$. Therapeutic APTT ranges defined by both this approach and the 1.5-2.5:control method are shown in Table 2. In each laboratory, the range defined by the anti-FXacorrelation method was broader than that defined using the 1.5-2.5:control method, and extended more than $29 \mathrm{~s}$ beyond the upper limit of the 1.5-2.5:control range.

\section{Interlaboratory correlations}

Figure 2 depicts interlaboratory correlation for the FXa inhibition assay for each pairwise comparison among the four laboratories, and Fig. 3 shows the interlaboratory correlation for the APTT assay for the same pairwise comparisons. The FXa inhibition assay demonstrated superior interlaboratory correlation to the APTT assay for all but one of the pairwise comparisons (laboratory A vs. laboratory B), but the degree of correlation for the FXa inhibition assay was nonetheless

Table 1 Reagents and instruments

\begin{tabular}{|c|c|c|c|c|}
\hline \multirow[b]{2}{*}{ Laboratory } & \multicolumn{2}{|l|}{ APTT } & \multicolumn{2}{|l|}{ Factor Xa inhibition } \\
\hline & Reagent & Instrument & Reagent & Instrument \\
\hline A & MDA Platelin L* & MDA-180* & Stachrom Heparin $\dagger$ & Coag-A-Mate MTX II* \\
\hline B & HemosIL SynthASil $\ddagger$ & ACL TOP $\ddagger$ & HemosIL Heparin $\ddagger$ & ACL TOP \\
\hline $\mathrm{C}$ & Actin FSL§ & BCS§ & Berichrome Heparin§ & BCS§ \\
\hline $\mathrm{D}$ & MDA Platelin L* & MDA-180* & Stachrom Heparin $\dagger$ & STA Compact $\dagger$ \\
\hline
\end{tabular}

APTT, activated partial thromboplastin time.

*bioMérieux (Durham, NC, USA). †Diagnostica Stago (Asnieres-sur-Seine, France). †Instrumentation Laboratory (Paris, France). §Dade Behring (Marburg, Germany). 

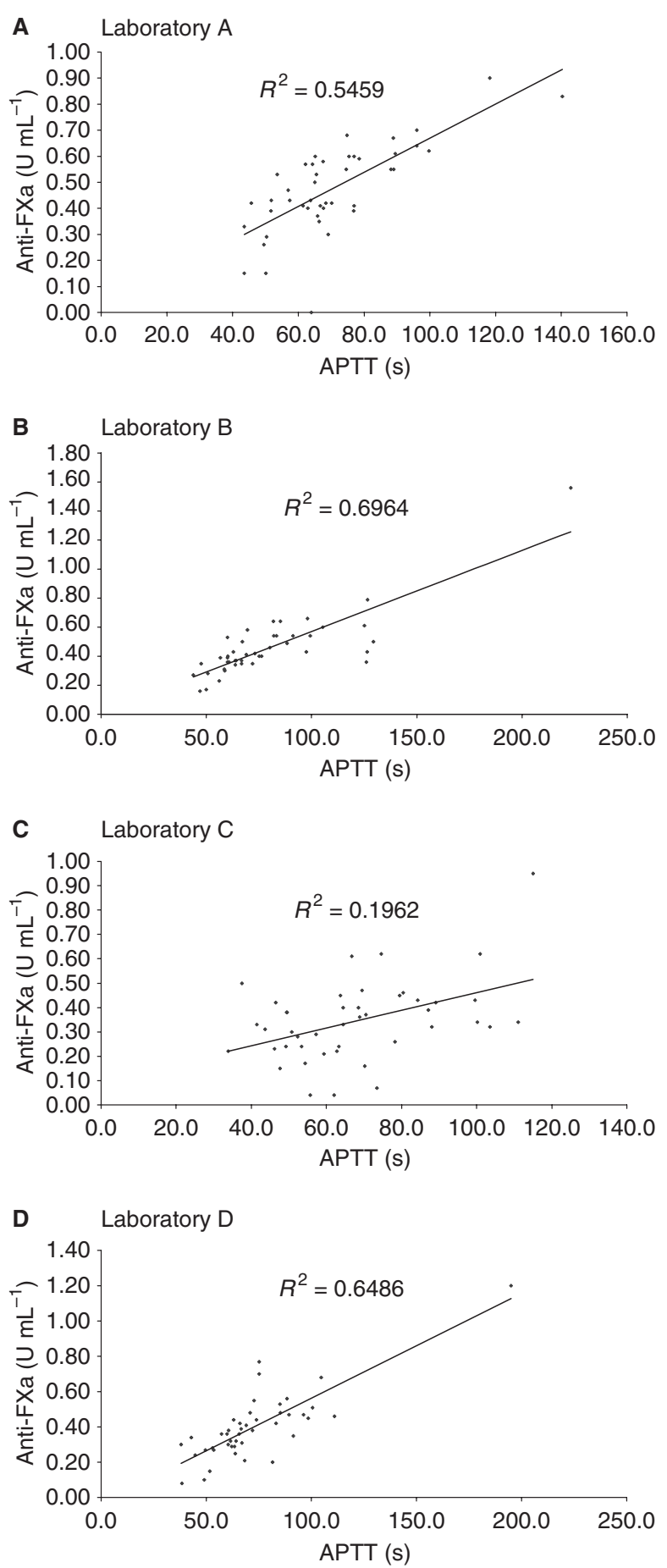

Fig. 1. Intralaboratory correlation between the activated partial thromboplastin time (APTT) and FXa inhibition assays. (A-D) Relationship between the APTT and FXa inhibition assays (anti-FXa) in each laboratory. The coefficient of determination $\left(R^{2}\right)$ is shown for each correlation.

modest, with the $R^{2}$ exceeding 0.8 in just one of the six pairwise comparisons. Interestingly, although laboratories $\mathrm{A}$ and $\mathrm{D}$ employed the same reagent in their FXa inhibition assay, and the same reagent and instrument in their APTT assay (Table 1), the correlation between them was nonetheless limited for both the anti-FXa assay (Fig. 2C) and APTT assay (Fig. 3C).
Table 2 Therapeutic activated partial thromboplastin time (APTT) ranges

\begin{tabular}{llll}
\hline & & \multicolumn{2}{l}{ Therapeutic APTT range (s) } \\
\cline { 3 - 4 } Laboratory & $\begin{array}{l}\text { APTT } \\
\text { reference } \\
\text { range (s) }\end{array}$ & $\begin{array}{l}\text { 1.5-2.5: } \\
\text { control } \\
\text { method }\end{array}$ & $\begin{array}{l}\text { Anti-FXa- } \\
\text { correlation } \\
\text { method }\end{array}$ \\
\hline A & $21.8-32.5$ & $40.7-67.9$ & $45.4-106.0$ \\
B & $27.0-37.1$ & $48.1-80.1$ & $53.6-125.0$ \\
C & $23.3-30.2$ & $40.1-66.9$ & $83.2-194.3$ \\
D & $24.0-37.0$ & $45.8-76.3$ & $61.7-105.4$ \\
\hline
\end{tabular}

The 1.5-2.5:control method ranges are derived by multiplying the midpoint of the APTT reference range by 1.5 and 2.5 , respectively. The anti-FXa-correlation method ranges are derived from the linear regressions shown in Fig. 1, such that the lower and upper limits of the therapeutic range correspond to 0.3 and 0.7 anti-FXa units $\mathrm{mL}^{-1}$, respectively.

\section{Analysis by therapeutic range}

Samples were segregated into therapeutic categories [i.e. below therapeutic (BT), therapeutic (T), or above therapeutic (AT)] on the basis of the laboratory-specific therapeutic APTT ranges shown in Table 2. Interlaboratory agreement with respect to therapeutic category for the 1.5-2.5:control method and the anti-FXa-correlation method was assessed. When the 44 samples were classified by therapeutic category using the 1.52.5:control method, $23(52 \%)$ showed agreement among all four laboratories, $17(39 \%)$ showed agreement among three laboratories, and four $(9 \%)$ showed agreement between only two laboratories. In contrast, when the samples were categorized by the laboratory-specific anti-FXa-derived APTT range, there was consensus among all four laboratories for just seven (16\%) samples, whereas $26(59 \%)$ showed agreement among three laboratories and $11(25 \%)$ showed agreement among only two laboratories. Three (7\%) samples were found to be simultaneously BT and AT in different laboratories with the anti-FXa-correlation method, whereas two (5\%) samples showed this discrepancy with the 1.5-2.5:control method.

Because the intralaboratory correlation between the APTT assay and FXa inhibition assay was particularly poor in laboratory C (Fig. 1C), analysis by therapeutic range was repeated with omission of this laboratory's results. When data from laboratory $\mathrm{C}$ were excluded, the anti-FXa-correlation method continued to show no apparent benefit over the 1.52.5:control method with respect to interlaboratory agreement. Specifically, therapeutic category agreement between laboratories $\mathrm{A}, \mathrm{B}$ and $\mathrm{D}$ was observed for $27(61 \%)$ samples with the 1.5-2.5:control method and $25(57 \%)$ samples with the antiFXa-correlation method.

The extent to which the poor interlaboratory agreement observed with the anti-FXa-correlation method was due to interlaboratory variation in the FXa inhibition assay itself was examined. Using the CAP's recommended anti-FXa therapeutic range for UFH of $0.3-0.7$ units $\mathrm{mL}^{-1}$, samples were categorized as $\mathrm{BT}\left(<0.3\right.$ units $\left.\mathrm{mL}^{-1}\right), \mathrm{T}\left(0.3-0.7\right.$ units $\left.\mathrm{mL}^{-1}\right)$, or AT (>0.7 units $\mathrm{mL}^{-1}$ ). Twenty-eight $(64 \%)$ samples 

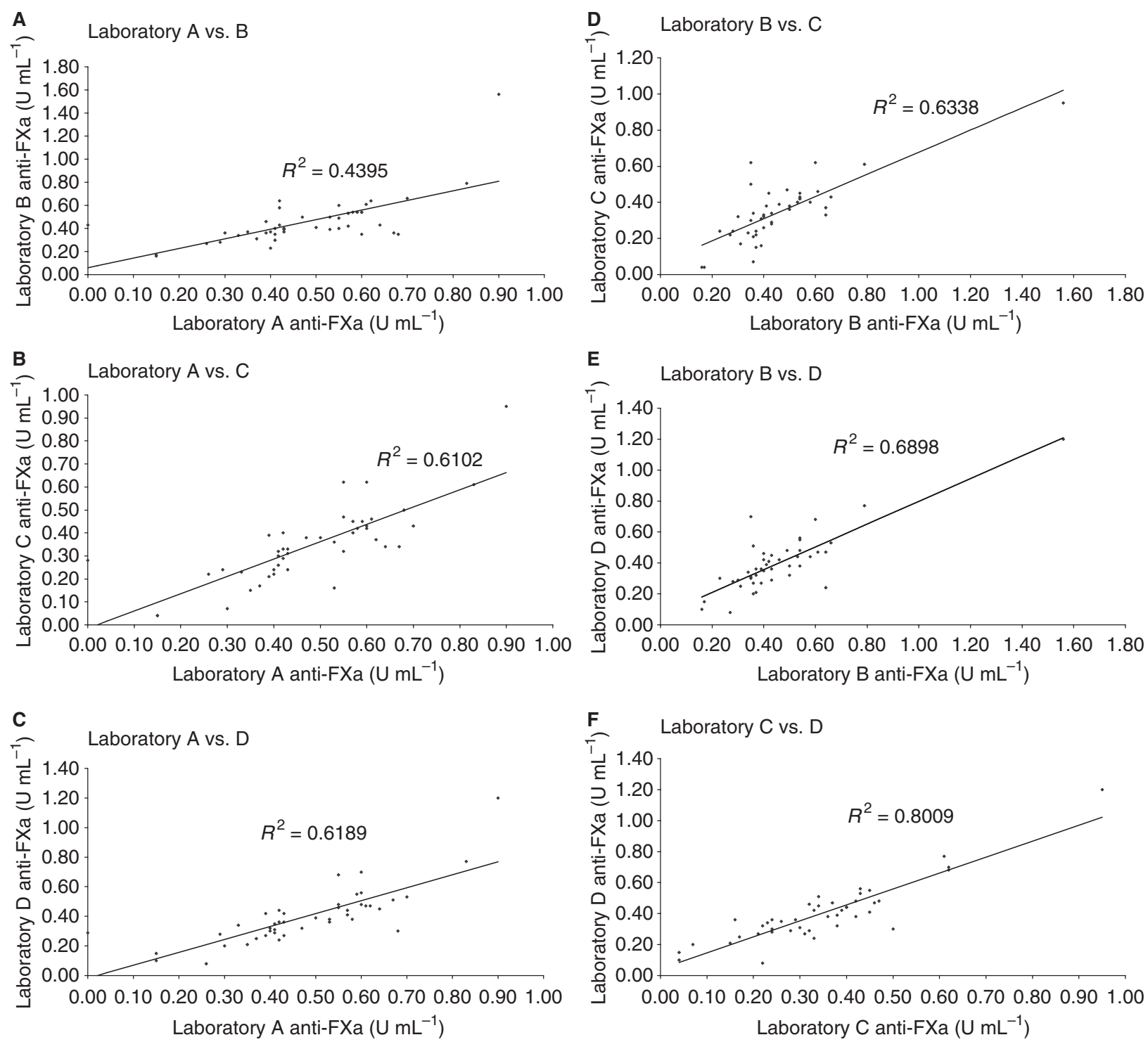

Fig. 2. Interlaboratory correlation for the FXa inhibition assay. (A-F) Pairwise correlations for the FXa inhibition assay (anti-FXa) among each of the four laboratories. The coefficient of determination $\left(R^{2}\right)$ is shown for each comparison.

showed therapeutic range agreement among all four laboratories, $11(25 \%)$ showed agreement among three laboratories, and five $(11 \%)$ showed agreement among only two laboratories. None of the 44 samples was found to be simultaneously AT and BT in different laboratories. When the data from laboratory $\mathrm{C}$ were excluded, therapeutic range agreement was observed among laboratories A, B and D for $35(80 \%)$ samples.

\section{Shipment analysis}

In order to determine whether transport and shipping conditions contributed to the observed variation among laboratories in the APTT and FXa inhibition assays, a simulated shipment experiment was performed. No significant differences in APTT or anti-FXa values were seen when samples analyzed before and after simulated shipment were compared (data not shown).

\section{Discussion}

Since 1998, the CAP has mandated that the therapeutic APTT range for UFH be defined in each laboratory through correlation with a direct measure of heparin activity such as the FXa inhibition assay [9], in part to improve upon the substantial interlaboratory variation observed with the traditional 1.5-2.5:control method. Whether the anti-FXacorrelation method satisfies this intent has not been reported. We performed a cross-validation study among four CAPaccredited specialized coagulation laboratories to compare the interlaboratory variation of the anti-FXa-correlation method with that of the traditional 1.5-2.5:control method.

In our small study, we observed significant interlaboratory variation in the anti-FXa-correlation method with respect to therapeutic range. With this method, there was consensus among the four laboratories as to whether a patient was BT, T 


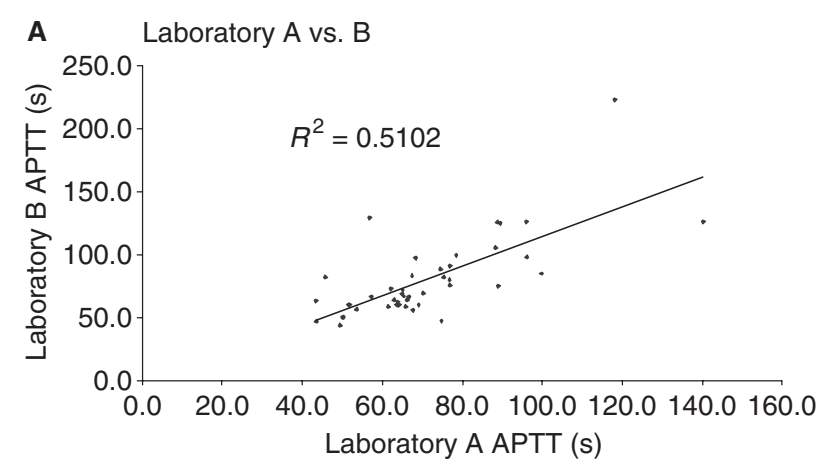

D Laboratory B vs. C

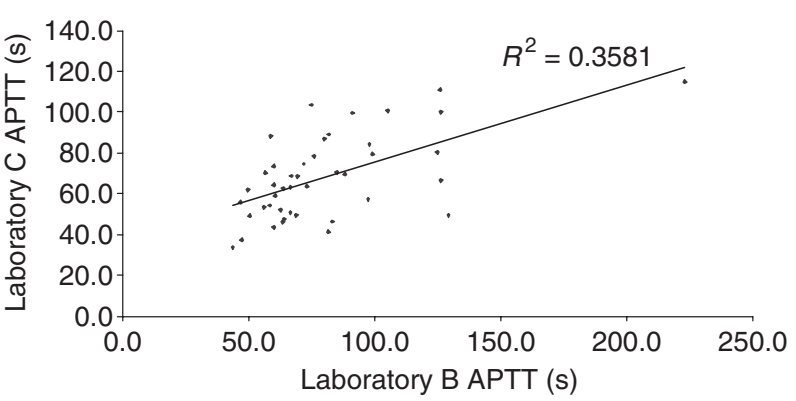

E Laboratory B vs. D

B Laboratory A vs. C
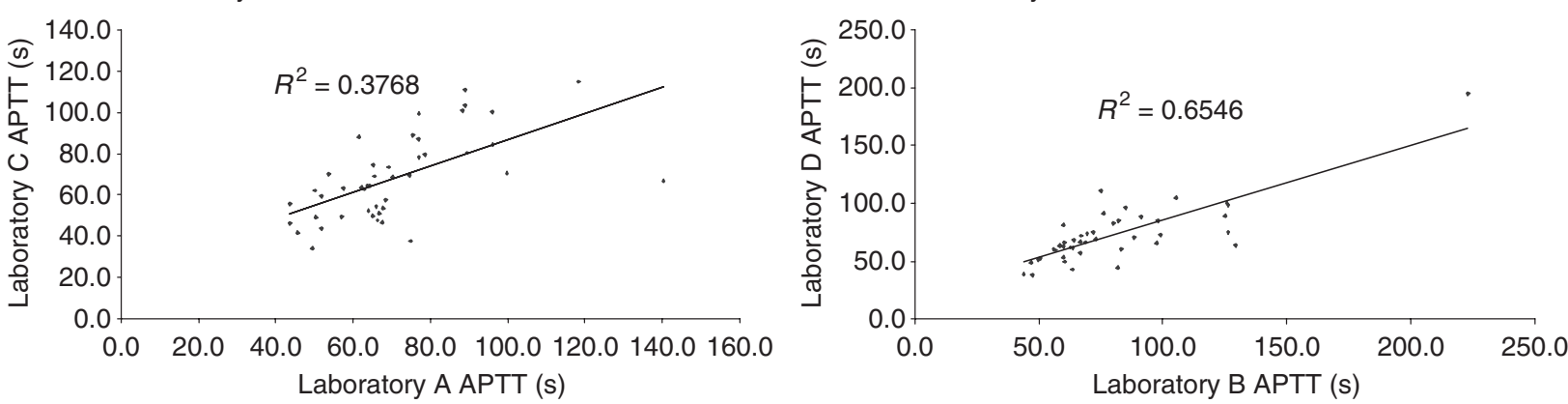

F Laboratory C vs.D

C Laboratory A vs. D

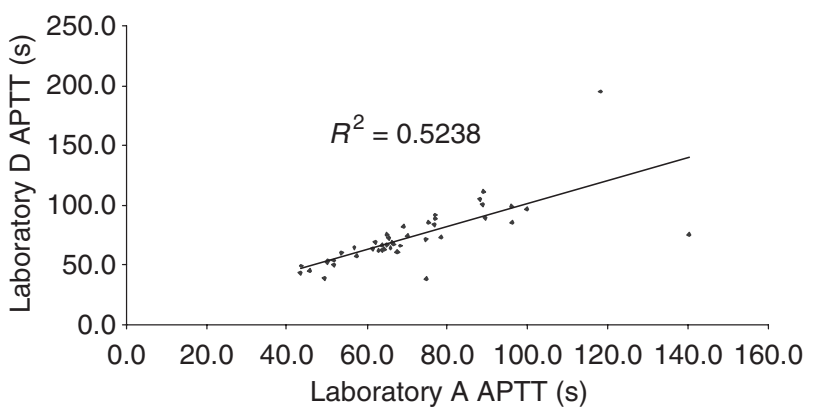

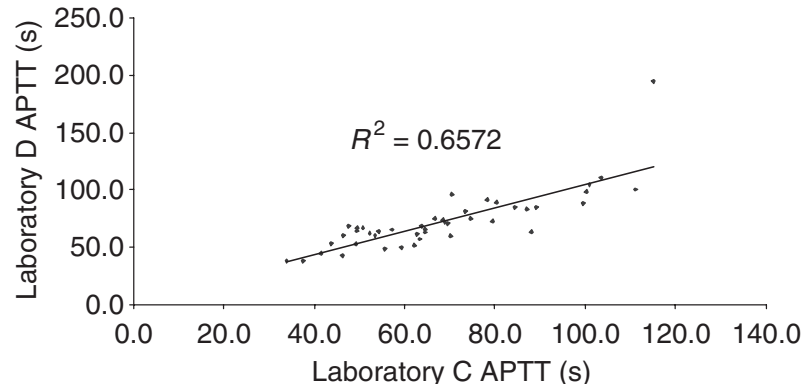

Fig. 3. Interlaboratory correlation for the activated partial thromboplastin time (APTT). (A-F) Pairwise correlations for the APTT assay among each of the four laboratories. The coefficient of determination $\left(R^{2}\right)$ is shown for each comparison.

or AT in only $16 \%$ of samples. In contrast, consensus was achieved in $52 \%$ of samples with the 1.5-2.5:control method. When consensus was redefined as therapeutic range agreement among at least three laboratories, it was achieved in $75 \%$ of samples with the anti-FXa-correlation method and in $91 \%$ of samples with the 1.5-2.5:control method. The anti-FXacorrelation method remained non-superior to the 1.5-2.5:control method with respect to interlaboratory consensus ( $57 \%$ vs. $61 \%$ ) when the poorly correlated data from laboratory $\mathrm{C}$ (Fig. 1C) were excluded from the analysis.

By definition, the validity of the anti-FXa-correlation method relies on two relationships - the intralaboratory correlation between the FXa inhibition assay and the APTT assay, and the interlaboratory correlation for the FXa inhibition assay. In our study, correlation for both of these relationships was modest. Consistent with previous analyses [11-13], we observed $R^{2}$ values of $0.5459-0.6964$ for the intralaboratory correlation between the anti-FXa and APTT assays in laboratories A, B and D (Fig. 1). However, laboratory $\mathrm{C}$ demonstrated an $R^{2}$ for this relationship of 0.1962 , substantially lower than what has been reported previously. Although assessments of correlation in the literature may be mildly inflated by publication bias, the very poor correlation between the APTT and anti-FXa assays in laboratory $\mathrm{C}$ raises concern about an unidentified technical problem with one or both of the assays. For this reason, therapeutic range analyses were performed both with and without the data from laboratory $\mathrm{C}$ as described above. Although the poor intralaboratory correlation observed in laboratory $\mathrm{C}$ constitutes a limitation of our study, it also highlights the technical challenges associated with the APTT and anti-FXa assays, an issue of real-world relevance to the coagulation laboratories in which these assays are performed. 
Modest interlaboratory correlation for the FXa inhibition assay was also observed, with the $R^{2}$ for the pairwise comparisons ranging from 0.4395 to 0.8009 (Fig. 2). These results are consistent with previously published reports, which, much as is observed with the APTT assay, show variation in the responsiveness of the FXa inhibition assay depending on the choice of reagent and instrumentation [14-16]. Interestingly, the correlation for the anti-FXa assay between laboratories A and D was limited (Fig. 2C), despite the use of a common reagent (Table 1). This may underscore the importance of instrument, as well as reagent, in the responsiveness of the anti-FXa assay to UFH [15]. Despite the use of a common reagent and a common coagulometer, limited correlation for the APTT assay between these centers (Fig. 3C) was also observed. This may reflect the laboratories' use of different reagent lots in their APTT assays, a variable that has also been reported to influence the response of the APTT to UFH [8]. Issues related to specimen storage and transport have been demonstrated to affect the APTT and FXa inhibition assays $[17,18]$, and represent another potential source of interlaboratory variation in our study. To address this possibility, a simulated shipment experiment was carried out; this showed no significant difference between the pre-shipment and postshipment APTT or anti-FXa, suggesting that the interlaboratory variation observed in our study is not attributable to preanalytical transport and shipping variables.

In spite of the modest interlaboratory correlation observed with the FXa inhibition assay, it showed somewhat better interlaboratory agreement than either the anti-FXa-correlation method or the 1.5-2.5:control method. Using the FXa inhibition assay, therapeutic range consensus among all four and among at least three laboratories was observed for $64 \%$ and $89 \%$ of samples, respectively. When the data from laboratory $\mathrm{C}$ were excluded, therapeutic range agreement among laboratories A, B and D was observed for $80 \%$ of samples. Furthermore, whereas several patient samples were found to be simultaneously AT and BT in different laboratories by both the anti-FXa-correlation method and the 1.5-2.5:control method, this striking discrepancy was not observed with the FXa inhibition assay itself.

These results suggest that the FXa inhibition assay may prove clinically superior to both the anti-FXa-correlation method and the 1.5-2.5:control method in the monitoring of UFH. However, enthusiasm for the FXa inhibition assay for this purpose has been tempered, both because of its greater cost than the APTT assay and because of clinicians' greater familiarity and comfort with the APTT assay. Furthermore, few clinical data are available to support use of the FXa inhibition assay in UFH monitoring. In a trial by Levine et al., patients with acute venous thromboembolism requiring large daily doses of UFH randomized to monitoring by either direct anti-FXa level or protamine titration-correlated APTT showed roughly equal rates of recurrent thrombosis and bleeding [19]. Because this study was performed within a single center, interlaboratory variation in the monitoring methods could not be assessed. To our knowledge, no other randomized trials of
UFH monitoring have been performed, and opinions on the clinical value of various monitoring techniques have been derived largely from observational and laboratory-based data. Clearly, randomized, controlled, multicenter clinical trials would be necessary to determine the optimal approach for UFH monitoring.

As UFH enters its eighth decade of clinical use [20], the optimal method for its monitoring remains unknown. Our small study suggests that the anti-FXa-correlation method, recommended by the CAP and ACCP for the purpose of improving upon the poor interlaboratory agreement observed with the 1.5-2.5:control method, does not appear to satisfy this intent. Further multicenter cooperative studies are required to confirm these findings. On the basis of our data, adoption of the anti-FXa-correlation method would appear to lead to great disparity in UFH dosing decisions among different centers. The clinical impact of this disparity is unknown and also warrants further investigation.

\section{Acknowledgement}

This study was funded in part by T32 HL07971 and a grant from the University of Pennsylvania Institute for Translational Medicine and Therapeutics (to A. Cuker and D.B. Cines); by a grant from the Chair's Fund of B. Wolf, Pathologist in Chief at Children's Hospital of Philadelphia (to X.L. Zheng); and by HL084006 and HL081012 (to D.B. Cines). We also wish to thank S. Gay, C. Sohier and M. Tanzer for their technical assistance, and C. Abrams and the anonymous reviewers for their insightful comments.

\section{Disclosure of Conflict of Interests}

The authors state that they have no conflict of interest.

\section{References}

1 Chiu HM, Hirsh J, Yung WL, Regoeczi E, Gent M. Relationship between the anticoagulant and antithrombotic effects of heparin in experimental venous thrombosis. Blood 1977; 49: 171-84.

2 Basu D, Gallus A, Hirsh J, Cade J. A prospective study of the value of monitoring heparin treatment with the activated partial thromboplastin time. N Engl J Med 1972; 287: 324-7.

3 Shapiro GA, Huntzinger SW, Wilson JE. Variation among commercial activated partial thromboplastin time reagents in response to heparin. Am J Clin Pathol 1977; 67: 477-80.

4 Kitchen S, Jennings I, Woods TA, Preston FE. Wide variability in the sensitivity of APTT reagents for monitoring of heparin dosage. J Clin Pathol 1996; 49: 10-14.

5 D'Angelo A, Seveso MP, D'Angelo SV, Gilardoni F, Dettori AG, Bonini P. Effect of clot-detection methods and reagents on activated partial thromboplastin time (APTT). Implications in heparin monitoring by APTT. Am J Clin Pathol 1990; 94: 297-306.

6 Kitchen S, Preston FE. The therapeutic range for heparin therapy: relationship between six activated partial thromboplastin time reagents and two heparin assays. Thromb Haemost 1996; 75: 734-9.

7 Bates SM, Weitz JI, Johnston M, Hirsh J, Ginsberg JS. Use of a fixed activated partial thromboplastin time ratio to establish a therapeutic range for unfractionated heparin. Arch Intern Med 2001; 161: 385-91. 
8 Brill-Edwards P, Ginsberg JS, Johnston M, Hirsh J. Establishing a therapeutic range for heparin therapy. Ann Intern Med 1993; 119: 104 9.

9 Olson JD, Arkin CF, Brandt JT, Cunningham MT, Giles A, Koepke JA, Witte DL. College of American Pathologists Conference XXXI on laboratory monitoring of anticoagulant therapy: laboratory monitoring of unfractionated heparin therapy. Arch Pathol Lab Med 1998; 122: 782-98.

10 Hirsh J, Bauer KA, Donati MB, Gould M, Samama MM, Weitz JI. Parenteral anticoagulants: American College of Chest Physicians Evidence-based clinical practice guidelines (8th edition). Chest 2008; 133: 141S-59S.

11 Toulon P, Boutiere B, Horellou MH, Trzeciak MC, Samama MM. Monitoring heparin therapy using activated partial thromboplastin time - results of a multicenter trial establishing the therapeutic range for SILIMAT, a reagent with high sensitivity to heparin. Thromb Haemost 1998; 80: 104-8.

12 Smythe MA, Koerber JM, Westley SJ, Nowak SN, Begle RL, Balasubramamiam M, Mattson JC. Use of the activated partial thromboplastin time for heparin monitoring. Am J Clin Pathol 2001; 115: 148-55.

13 Baker BA, Adelman MD, Smith PA, Osborn JC. Inability of the activated partial thromboplastin time to predict heparin levels. Time to reassess guidelines for heparin assays. Arch Intern Med 1997; 157: $2475-9$.
14 Kitchen S, Theaker J, Preston FE. Monitoring unfractionated heparin therapy: relationship between eight anti-Xa assays and a protamine titration assay. Blood Coagul Fibrinolysis 2000; 11: 137-44.

15 Kovacs MJ, Keeney M, MacKinnon K, Boyle E. Three different chromogenic methods do not give equivalent anti-Xa levels for patients on therapeutic low molecular weight heparin (dalteparin) or unfractionated heparin. Clin Lab Haematol 1999; 21: 55-60.

16 Ignjatovic V, Summerhayes R, Gan A, Than J, Chan A, Cochrane A, Bennett M, Horton S, Shann F, Lane G, Ross-Smith M, Monagle P. Monitoring unfractionated heparin (UFH) therapy: which antifactor Xa assay is appropriate? Thromb Res 2007; 120: 347-51.

17 van den Besselaar AM, Meeuwisse-Braun J, Jansen-Gruter R, Bertina RM. Monitoring heparin therapy by the activated partial thromboplastin time - the effect of pre-analytical conditions. Thromb Haemost 1987; 57: 226-31.

18 Adcock D, Kressin D, Marlar RA. The effect of time and temperature variables on routine coagulation tests. Blood Coagul Fibrinolysis 1998; 9: 463-70.

19 Levine MN, Hirsh J, Gent M, Turpie AG, Cruickshank M, Weitz J, Anderson D, Johnson M. A randomized trial comparing activated thromboplastin time with heparin assay in patients with acute venous thromboembolism requiring large daily doses of heparin. Arch Intern Med 1994; 154: 49-56.

20 Crafoord C. Preliminary report on post-operative treatment with heparin as a preventive of thrombosis. Acta Chir Scand 1937; 79: 407-26. 